\title{
COMPLEMENTARITY AND POTENTIAL OF POLSAR AND TOMOSAR FOR GLACIER SUBSURFACE CHARACTERIZATION
}

\author{
Giuseppe Parrella ${ }^{1}$, Georg Fischer ${ }^{1}$, Matteo Pardini ${ }^{1}$, Konstantinos Papathanassiou ${ }^{1}$, Irena Hajnsek ${ }^{1,2}$ \\ German Aerospace Center - Microwaves and Radar Institute, Oberpfaffenhofen, Germany \\ ETH Zurich - Institute of Environmental Engineering, Zurich, Switzerland
}

\begin{abstract}
Active microwave sensors, such as synthetic aperture radars (SARs), offer all-weather and daylight independent operability which is of great advantage for monitoring polar regions, where extreme environmental conditions and long period of darkness strongly limit other kinds of sensors. In addition, microwaves allow penetrating into dry snow and ice, making SAR measurements sensitive to the subsurface structure of glaciers and ice sheets. However, the retrieval of glacier subsurface parameters from SAR observations remains difficult due to their sensitivity to a large number of factors, including snow and ice properties, presence of layers, etc. The objective of this study is to attempt advancing the understanding of SAR measurements of glaciers and ice sheets. A combined analysis of polarimetric and tomographic measurements is carried out to derive a 3-D characterization of the scattering mechanisms occurring in a glacier subsurface scenario. The investigation exploits a fully-polarimetric tomographic airborne dataset, acquired over Greenland by the DLR's F-SAR system in the frame of the ARCTIC15 campaign.
\end{abstract}

Index Terms - One, two, three, four, five

\section{INTRODUCTION}

Remote sensing techniques represent nowadays a powerful tool for the monitoring of glaciers and ice sheets due to their capability to perform systematic and frequent observations of the polar regions. Among them, synthetic aperture radars (SARs) are able to operate also at unfavorable weather conditions and during the long dark polar winters. In addition, SARs benefit from the capability of microwaves to penetrate into dry snow and ice, gaining sensitivity to both surface and near-surface features of ice masses. This can be exploited to retrieve information about surface mass balance processes (e.g. refreezing of melt water) and subsurface ice properties. However, the retrieval of geophysical parameters from SAR measurements is rather complex due to their sensitivity to a large number of factors (e.g. snow and ice properties, presence and of melt layers, inclusions, etc.).
Polarimetric SAR (PolSAR) expands the observation space of single-channel SARs using polarization diversity to provide sensitivity to scattering mechanisms (volume, surface, anisotropic propagation, etc.). On the other hand, SAR tomography (TomoSAR) is based on multi-baseline (MB) interferometric measurements and allows retrieving the 3-D distribution of backscattering within volumes. The combination of the two techniques is, therefore, expected to provide a 3-D characterization of the scattering mechanisms occurring, for instance, in the subsurface.

This paper presents the analysis of a polarimetric and tomographic dataset acquired over the Greenland ice sheet exploiting the complementarity of the two techniques to improve the understanding of SAR measurements and their link to glacier subsurface features. The study focuses on P-band observations of the K-transect, in the ablation zone of the ice sheet, which were acquired in spring 2015 by the F-SAR airborne system of the DLR, during the ARCTIC15 campaign.

\section{STATE OF THE ART}

The interpretation of PolSAR signatures of glaciers and ice sheets has been addressed in several studies, mainly based on scattering models tailored to the respective test sites and employed frequencies [1][2][3]. A common limitation of such approaches relies in the limited observation space defined by polarimetry alone. In particular, PolSAR measurements are sensitive to the scattering mechanisms generated in each resolution cell along depth (within the penetration depth of the employed frequency) but do not provide the possibility to resolve the vertical distribution of the different contributions. Such limitation can be tackled by exploiting the interferometric dimension of the SAR observation space.

By using multi-baseline (MB) interferometric measurements, TomoSAR techniques can be adopted to retrieve the vertical distribution of backscattering. However, TomoSAR profiles are insensitive to non-scattering snow and firn layers, which can be instead detected with PolSAR by means of propagation effects resulting in co-polarization phase differences (CPD) [3]. The potential of TomoSAR for 


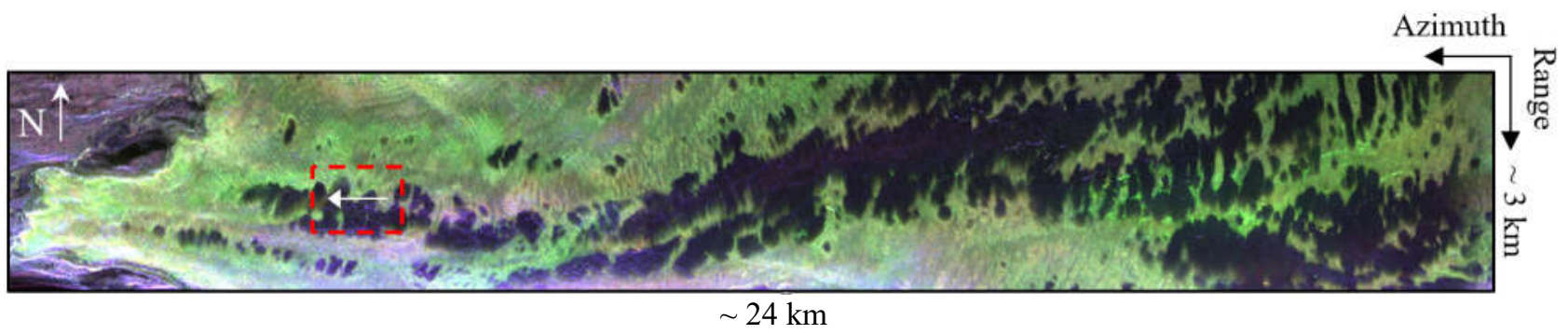

Figure 1: RGB representation of the P-band PolSAR data (R: HH-VV, G: 2HV, B: HH+VV).
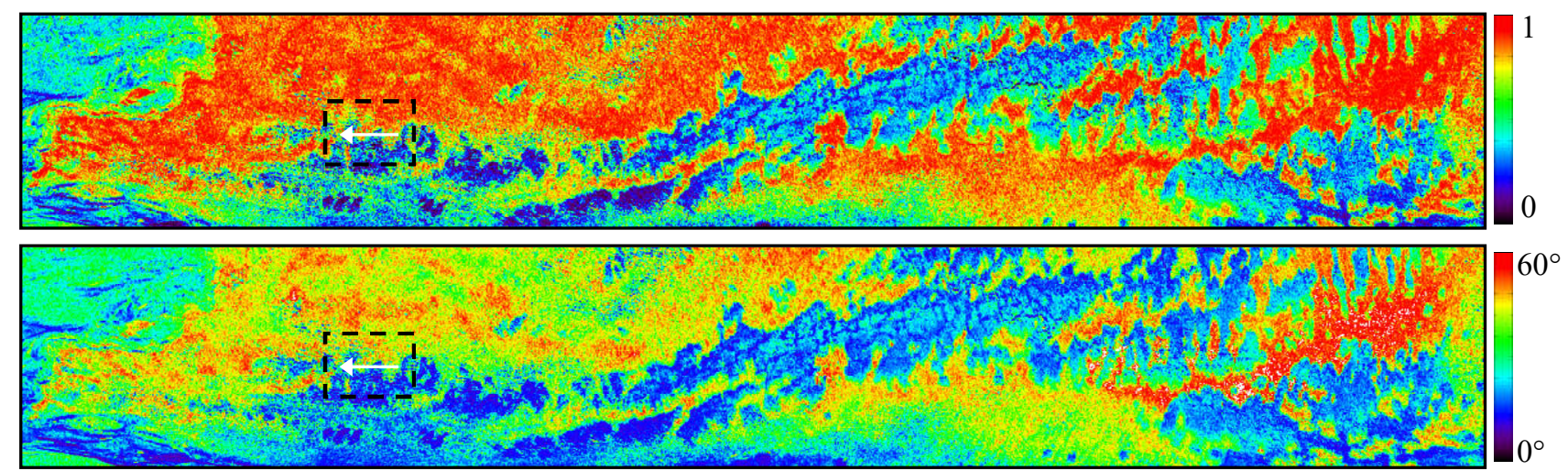

Figure 2: P-band polarimetric entropy (top) and mean alpha angle (bottom).
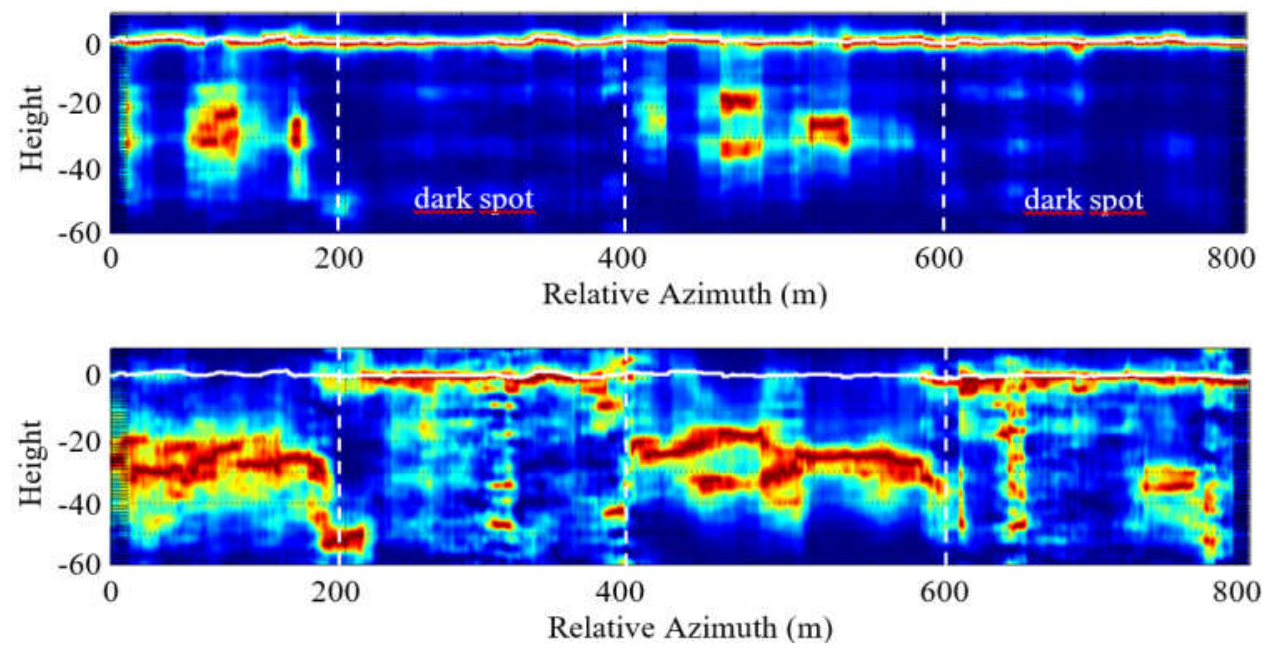

Figure 3: P-band tomographic profiles obtained in the HH (top) and HV (bottom) channel. The white line indicates the position of the surface from the external X-band DEM.

glacier subsurface imaging has been already shown in several airborne studies [4], [5], [6], [7]. Nevertheless, a physical link between TomoSAR observables and actual subsurface features has not been established yet, essentially due to the scarcity of suitable SAR and validation data.

\section{METHODOLOGY}

This study aims to assess the potential of combining polarimetric and tomographic SAR measurements for the characterization of glacier subsurface features. In particular, the complementarity of such techniques offers great 
potential to improve the current understanding of SAR measurements of ice masses. In this sense, a polarimetric analysis based on a set of descriptors is coupled with the retrieval of TomoSAR backscattering vertical profiles from a selected study area. The set of PolSAR descriptors includes the scattering entropy, the mean alpha angle, the CPD and polarimetric ratios, as proposed in [3]. In particular, the co-pol ratio is an indicator of the scatterers' orientation: values $>1$ suggest scattering from horizontal structures, values $\approx 1$ are attributed to a random volume, and values $<1$ indicate scattering from vertical features or from a (slightly rough) interface. The CPD provides information about the occurrence of anisotropic propagation through snow and firn layers, even in absence of scattering sources. The scattering entropy describes the complexity of the backscattering (in terms of the number of scattering mechanisms), and the mean alpha angle can be used to identify the type of the main scattering mechanism. In combination with the 3-D backscattering distribution profiles obtained from TomoSAR, such information potentially allows to achieve a complete 3-D characterization of the scattering scenario.

\section{PRELIMINARY RESULTS}

The test site is the so-called K-transect, in south-west Greenland. It is a regularly studied area of the ice sheet, with in-situ surface mass balance measurements since the early 1990s and a number of glaciological studies assessing different aspects of its temporal dynamics, which can support the SAR analysis. The available polarimetric and tomographic SAR dataset consists of P-band measurements covering an area of about $3 \mathrm{~km} \times 24 \mathrm{~km}$, from the west coast to the inner part of the ablation zone of the ice sheet. Figure 1 shows the polarimetric Pauli RGB representation of the SAR data, which provides first insights on the scattering mechanisms occurring in the area. In particular, a number of dark spots, aligned parallel to the azimuth direction, can be clearly identified in the mostly greenish background pixels which point out dominant volume scattering. In contrast, the dark features are characterized by strong surface-like scattering, as pointed out by the low scattering entropy and mean alpha angle values (see Figure 2). Despite the indication of two dominant scattering types, it remains difficult to understand to which extent surface and subsurface features contribute to the total backscattering. Figure 3 shows the vertical reflectivity profiles retrieved for the $\mathrm{HH}$ and $\mathrm{HV}$ channel along the $800 \mathrm{~m}$ long transect indicated in Figure 2 (white arrow in the dashed box). For convenience, the transect has been chosen to be at middle range and parallel to the azimuth direction. Being interested in the different polarimetric behavior observed for the 'dark spots' and the greenish background in the Pauli image, the TomoSAR transect has been selected to cross multiple transitions between such two features. Interestingly, the reflectivity profiles in Figure 3 clearly show the presence of two subsurface structure types, which correspond very well spatially to the areas of different polarimetric behaviors. The transect portions belonging to the greenish background of the Pauli image, characterized by dominant volumetric scattering, exhibit scattering contributions over a wide depth interval, down to $60 \mathrm{~m}$, with significant backscattering generated also at the surface in the $\mathrm{HH}$ channel. In contrast, the 'dark spots', exhibiting dominant surface-like scattering, are clearly dominated by scattering occurring at the surface, with relevant subsurface scattering only in the HV channel.

It appears already clear that the complementarity of PolSAR and TomoSAR has great potential for the characterization of the identified features. In this study, further analysis will be carried out to attempt establishing a link between the SAR data and the subsurface structure and dynamics ongoing in the test site. The results will be presented in the final paper.

\section{REFERENCES}

[1] E. J. Rignot, "Backscatter model for the unusual radar echoes from the Greenland ice sheet," J. Geophys. Res., 100(E5), 93899400, May 1995.

[2] J. J. Sharma, et al., "Polarimetric decomposition over glacier ice using long-wavelength airborne PolSAR," IEEE TGRS, 49(1), 519-534, Jan. 2011.

[3] G. Parrella, K. Papathanassiou and I. Hajnsek, "Polarimetric decomposition of L-band PolSAR backscattering over the Austfonna ice cap", IEEE TGRS, 54(3), 1267-1281, Mar. 2016.

[4] F. Banda, J. Dall, and S. Tebaldini, "Single and multipolarimetric P-band SAR tomography of subsurface ice structure," IEEE TGRS, 54(5), 2832-2845, May 2016.

[5] S. Tebaldini, T. Nagler, H. Rott, A. Heilig, "Imaging the internal structure of an Alpine glacier via L-band airborne SAR tomography”, IEEE TGRS, 54(12), 7197-7209, Dec. 2016.

[6] M. Pardini, G. Parrella, G. Fischer, K. Papathanassiou, "A multi-frequency SAR tomographic characterization of sub-surface ice volume", in Proc. of EUSAR'16, Berlin, Germany.

[7] G. Fischer, M. Jager, K. P. Papathanassiou, and I. Hajnsek, "Modeling the Vertical Backscattering Distribution in the Percolation Zone of the Greenland Ice Sheet With SAR Tomography," IEEE JSTARS, 12(11), Nov. 2019, doi: 10.1109/JSTARS.2019.2951026. 